\author{
Correspondencia
}

\title{
Colección Mastozoológica, Museo de Historia Natural UNILLANOS MHNU-M
}

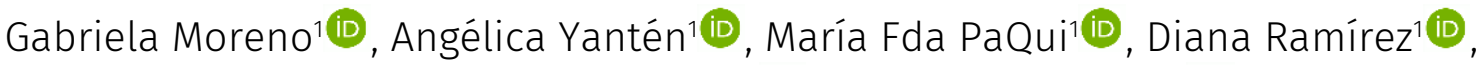 \\ Orlando Fabián Hernández ${ }^{1}$ (D) Francisco Sánchez ${ }^{* *}$ (i) \\ 1 Grupo de Investigación ECOTONOS, Programa de Biología, Facultad de Ciencias Básicas e Ingeniería, Universidad de los \\ Llanos, sede Barcelona, km 12 vía Puerto López, Villavicencio, Colombia. \\ * Correspondencia: fsanchezbarrera@unillanos.edu.co
}

\section{Resumen}

La Colección Mastozoológica del Museo de Historia Natural-Unillanos (MHNU-M) alberga en el momento 422 especímenes pertenecientes a ocho órdenes, 16 familias, 46 géneros, y 66 especies. Los especímenes provienen de cinco departamentos de Colombia, principalmente de la región de la Orinoquía. El número de especímenes de la Colección se ha enriquecido con actividades académicas de la Universidad de los Llanos, tesis de pre y posgrado, convenios y proyectos de investigación.

Palabras clave: Chiroptera, Colección biológica, Mamíferos, Orinoquía.

\begin{abstract}
The Mammalogy Collection of the Museum of Natural History of Unillanos (MHNU-M) is currently housing 422 specimens of eight orders, 16 families, 46 genera, and 66 species. The specimens come from five Colombian departments, mainly from the Orinoco region. The Collection has received material mostly from academic activities developed at the Universidad de los Llanos, undergraduate and graduate thesis, institutional agreements, and research projects.
\end{abstract}

Key words: Biological collection, Chiroptera, Mammals, Orinoco Region.

El Museo de Historia Natural de la Universidad de los Llanos fue reconocido legalmente como unidad académica por la misma Universidad en febrero del año 2018. En el Museo se aloja la Colección Mastozoológica con registro nacional de colecciones 255 de mayo de 2020. La Colección Mastozoológica (MHNU-M) se encuentra ubicada en Villavicencio-Meta (Figura 1), y ha recibido contribuciones principalmente de prácticas de campo de los cursos de Profundización en Fauna, y Sistemática/Taxonomía Animal del Programa de Biología. Los primeros especímenes fueron recolectados por los estudiantes de biología y por los docentes Gabriel Pantoja y Daniel Rodríguez en los años 2014 y 2015, respectivamente. Desde el segundo semestre del 2015, Francisco Sánchez ha estado a cargo de la colección y ha trabajado, junto con estudiantes del Semillero de Investigación Mamíferos Silvestres Unillanos en su organización y curatoría. Además, los integrantes del Semillero han sido fundamentales para ayudar en la preparación de especímenes. 


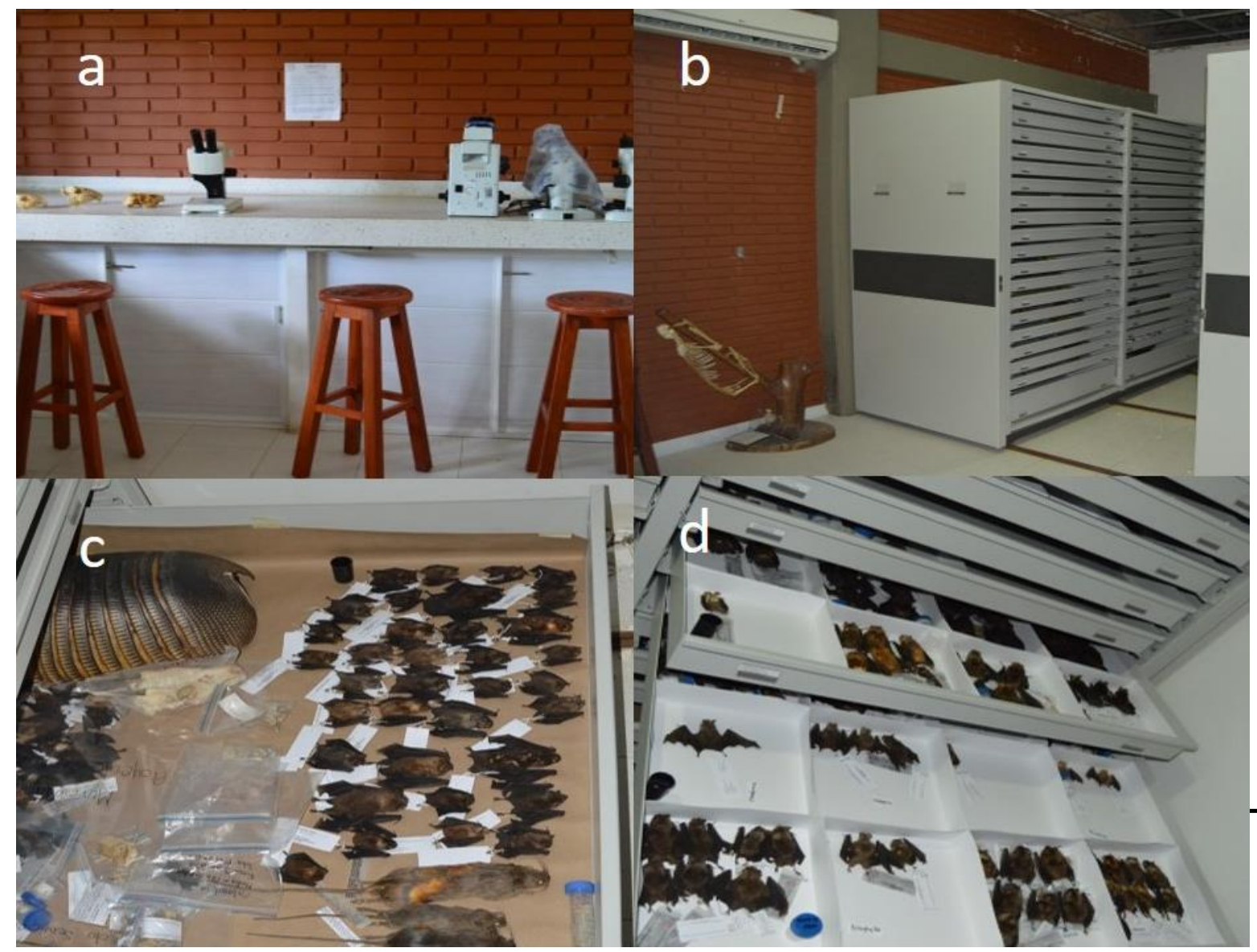

FIGURA 1. Instalaciones y ejemplares de la Colección Mastozoológica del Museo de Historia NaturalUnillanos (MHNU-M). a. Sala de revisión del Museo; b. Compactador para el almacenamiento de ejemplares; c y d. Ejemplares de la Colección.

Actualmente la colección del MHNU-M alberga 422 especímenes pertenecientes a ocho órdenes, 16 familias, 46 géneros, y 66 especies (Tabla 1). En la Colección hay especímenes de dos especies exóticas (Mus musculus y Rattus rattus) y 64 especies nativas, de las cuales hay dos especies endémicas de Colombia, Cryptotis thomasi y Plecturocebus ornatus (Woodman \& Péfaur 2007; APC 2016). El orden Chiroptera concentra el mayor número de especímenes (393), distribuidos en seis familias, 33 géneros y 53 especies. En términos de cobertura geográfica, la Colección ha recibido ejemplares provenientes principalmente de la Orinoquía colombiana, y en particular de los siguientes departamentos: Boyacá (2 municipios, 41 especimenes), Casanare (2 municipios, 129 especímenes), Cundinamarca (3 municipios, 15 especímenes), Meta (5 municipios, 225 especímenes) y Vichada (1 municipio, 12 especimenes).

La Colección ha apoyado proyectos de investigadores y estudiantes de pregrado de la Universidad de los Llanos y de un estudiante de postgrado de la Pontificia Universidad Javeriana (Alfonso 2017; Bernal 2018; Fino 2018; Ramírez-Mejía 2017; Ramírez 2018). También ha prestado sus servicios en proyectos de investigación en conjunto con organizaciones como Wildlife Conservation Society y The Nature Conservancy. Producto de estos esfuerzos de investigación se ha logrado, hasta el momento, la publicación de cinco trabajos: 


(2019), Alfonso \& Sanchez

Sánchez (2017), Cruz-Parrado et al. (2018), Pérez-García et al. (2019), Alfonso \& Sánchez (2019), y Ramírez-Mejía et al. (2020).

TABLA 1. Número de órdenes, familias, géneros y especies depositadas en la Colección Mastozoológica del Museo de Historia Natural-Unillanos (MHNU-M).

\begin{tabular}{lcccc}
\hline \multicolumn{1}{c}{ Órdenes } & Familias & Géneros & Especies & Número de especímenes \\
\hline Didelphimorphia & 1 & 3 & 3 & 4 \\
Cingulata & 1 & 1 & 1 & 1 \\
Pilosa & 1 & 1 & 1 & 1 \\
Eulipotyphla & 1 & 1 & 1 & 3 \\
Chiroptera & 6 & 33 & 53 & 393 \\
Carnivora & 1 & 1 & 1 & 1 \\
Primates & 2 & 2 & 2 & 3 \\
Rodentia & 3 & 4 & 4 & 16 \\
Total & 16 & 46 & 66 & 422 \\
\hline
\end{tabular}

Adicionalmente, un convenio entre la Sociedad Colombiana de Mastozoología y el Instituto Alexander von Humboldt, facilitó una beca para D. Ramírez con el fin de organizar especímenes de la Colección y cargar datos en el Sistema de Información sobre Biodiversidad de Colombia SIB-Colombia. Esta información se encuentra disponible en los siguientes enlaces: http:/ /ipt.biodiversidad.co/sib/resource?r=registros-mhnu-unillanos http:/ / www.gbif.org/dataset/ efedb425-09ae-4220-831b-51f7b1ab48eb

Hasta la fecha, la Colección del MHNU-M, ha sido un espacio de aprendizaje para los estudiantes del Programa de Biología de Unillanos. La Colección ofrece la oportunidad de aprender y desarrollar actividades que promueven la formación académica de jóvenes biólogos, sobre todo al incrementar el interés por el estudio e investigación de los mamíferos neotropicales. Esperamos que en el futuro la Colección pueda ser aprovechada por otros interesados en los mamíferos además de los miembros de Unillanos. La Colección de Unillanos es la única en la Orinoquía registrada actualmente ante el Instituto Alexander von Humboldt, y espera convertirse en un referente de la diversidad de los mamíferos de la región, para apoyar su investigación y conservación.

\section{AGRADECIMIENTOS}

El funcionamiento de la Colección es posible gracias al apoyo de la Universidad de los Llanos y su Facultad de Ciencias Básicas e Ingeniería. Gracias a Andrea Morales por los comentarios al documento. A la Sociedad Colombiana de Mastozoología por la invitación y a Héctor Ramírez-Chaves por el "empujoncito" para escribir esta nota y por la revisión de la misma. Esta es la publicación número 009 del Museo de Historia Natural-Unillanos. 


\section{REFERENCIAS}

APC, Asociación Primatologíca Colombiana. 2016. Lista de primates colombianos. https://www.asoprimatologicacolombiana.org/primates-de-colombia.html. Consultado 2 julio 2020.

Alfonso AF. 2017. Mamíferos terrestres de un campus universitario en el piedemonte llanero colombiano y evaluación de cebos para su captura: Programa de Biología, Universidad de los Llanos, Villavicencio.

Alfonso A, Sánchez F. 2019. Mamíferos no voladores en un campus universitario de la Orinoquia colombiana. Mammalogy Notes. 5(2):29-39. https://doi.org/10.47603/manovol5n2.29-39

Bernal KA. 2018. Uso del espacio por murciélagos insectívoros en un gradiente urbano-rural en Villavicencio-Meta: Programa de Biología, Universidad de los Llanos, Villavicencio.

Cruz-Parrado K, Moreno G, Sánchez F. 2018. Dieta de Saccopteryx leptura (Chiroptera: Emballonuridae) en un área exurbana del piedemonte llanero colombiano. Revista Facultad de Ciencias Básicas. 1(1):138-142. https:// doi.org/10.18359/rfcb.3255

Fino E. 2018. Murciélagos filostómidos consumidores de insectos en un paisaje urbano-rural del piedemonte llanero colombiano: Programa de Biología, Universidad de los Llanos, Villavicencio

Pérez-García C, Bernal-Contreras K, Ramírez-Castellanos DM, Buitrago-Valenzuela DC, CeballosLadino LA, Sánchez-Barrera F. 2019. Edificios usados como refugios por murciélagos en un campus universitario del piedemonte llanero de Colombia. Orinoquia. 23(2):109-120. https:// doi.org/10.22579/20112629.574

Ramírez-Mejía A. 2017. Diversidad funcional, taxonómica y dispersión de semillas por murciélagos filostómidos en un paisaje antropizado: un análisis multi-escala: Maestría en Conservación y Uso de la Biodiversidad, Pontificia Universidad Javeriana, Bogotá.

Ramírez-Mejía AF, Urbina-Cardona JN, Sánchez F. 2020. Functional diversity of phyllostomid bats in an urban-rural landscape: A scale-dependent analysis. Biotropica 2020; 00:1- 15. https:// doi.org/10.1111/btp.12816

Ramírez D. 2018. Presas Potenciales de los murciélagos insectívoros Molossus molossus (Molossidae) y Saccopteryx leptura (Emballonuridae) en Villavicencio - Meta: Programa de Biología, Universidad de los Llanos, Villavicencio.

Sánchez F. 2017. Bats of Villavicencio (Meta, Colombia): Preliminary assessment of their trophic diversity and ecosystem services. Boletín Científico del Centro de Museos de Historia Natural. 21(1):96-111.

Woodman N, Péfaur J. 2007. Order Soricomorpha Gregory, 1910. In: Gardner AL, Editor. Mammals of South America. Volume I: marsupials, xenarthrans, shrews, and bats. Chicago, USA. University of Chicago Press. p. 177-187.

Editor: Héctor E. Ramírez-Chaves

Recibido: 2020-07-08

Revisado: $2020-07-10$

Aceptado: 2020-07-20

Publicado: 2020-10-04 